\title{
PROFILES OF ICF DISABILITY IN ALZHEIMER AND VASCULAR DEMENTIA
}

\author{
A. Malara, G. Sgrò, F. Ceravolo, G. Curinga, G.F. Renda, F. Spadea, V. Rispoli
}

\begin{abstract}
Backgrounds: The International Classification of Functioning, Disability and Health (ICF) is a suitable tool to standardize the status of health and disability. A previous study, carried out on 546 subjects included in the database ANASTE (National Association of Nursing Home for Third Age) Calabria, showed that $78.43 \%$ of the patients suffered from cognitive impairment whereas $52 \%$ had a severe degree of dementia. $65 \%$ of them was suffering from Alzheimer's Disease (AD), whereas $23 \%$ from vascular dementia (VD). Objectives: Aim of this study was to analyse the prevalence of functional impairments, activity limitations and participation restrictions of patients affected by AD and VD. Design: Observational descriptive study. Setting: Nursing Homes ANASTE Calabria. Participants: 10 patients with probable AD (ADPr) and 10 patients affected by probable VD (VDPr). Measurements: All patients were underwent multidimensional geriatric assessment. The profiles of disability ICF, were expressed in terms of Capacity and Performance, and coded according to mild, medium, severe and complete disability. Environmental factors were skilled in facilitator or no facilitator. Results: The patients with ADPr displayed a severe impairment of functional status, and advanced clinical stage requiring higher care burden compared with VDPr patients. The ICF assessment showed that the global and specific Mental Functions, Communication and Interpersonal Relationships were more reduced in patients with ADPr respect those with VDPr. Conclusions: The identification of a ICF checklist of various forms of dementia, might provides a more detailed description of the profiles of disability and improving therapeutic, rehabilitative interventions and psico-social care.
\end{abstract}

Key words: ICF, dementia, nursing home.

\section{Introduction}

The International Classification of Functioning, Disability and Health (ICF) was published by the World Health Organization (WHO) in 2001 to standardize descriptions of health and disability (1). ICF organizes information in three components:

- The Body construct, which comprises two classifications, one for body functions, and another for body structure. Body functions and body structures refer to the human organism as a whole, it includes the brain and its functions, (i.e. the mind).

- The Activities and Participation constructs denote the aspects of functioning from both an individual and social perspective. The domains of activity and participation are listed in a single list that includes the large range of areas of life, by learning basic to social tasks.

- The Environmental factors make up the physical environment, social environment in which people live

Scientific Commettee of National Association of Nursing Home for Third Age (ANASTE) Calabria, Lamezia Terme (CZ), Italy

Corresponding Author: Alba Malara, Scientific Commettee of National Association of Nursing Home for Third Age (ANASTE) Calabria, Lamezia Terme (CZ), Italy E-mail: alba.doc@tiscali.it, mobile phone: +39.340.6621250, fax: +39.0968.400478 and conduct their lives. The factors are external to individuals and can have a positive or negative effect on participation, activities or on the individual's body function or structure. The Personal factors are the individual background of an individual's life, regardless of their health condition. These may include age, race, sex, education, experience, personality and character style, aptitudes, fitness, lifestyle, education, coping styles, social background, profession (2).

Each of the components can be expressed in terms of both positive and negative influence. The negative aspects are defined as «impairment» (dysfunction or loss of BF), «limitation of Activity «(individual difficulty in performing a particular activity) or «restriction of Participation» (individual problems in involvement in life situations) (3). Impairments of structure can involve an anomaly, defect, loss or other significant deviation in body structures. Impairments can be temporary or permanent; progressive, regressive or static; intermittent or continuous. Difficulties or problems of these domains can arise when there is a qualitative or quantitative alteration in the way in which the functions of these domains are carried out. These characteristics are captured in further descriptions, mainly in the 
codes, by means of qualifiers. The positive aspects are expressed as AP codes, that identify profiles of mild, serious and complete disability, expressed in terms of «Capacity» and «Performance». The Capacity qualifier describes the highest level of functioning of a person to perform a task; the Performance describes what a person does, in actual conditions, considering all available environmental factors (instrumental and personal). Differences between Capacity and Performance qualifiers indicate the presence of environmental factors that facilitate or hinder the operating profile (1). ICF is a multi-purpose classification designed to serve various disciplines and different sectors. Its specific aim is to provide a scientific basis for understanding and studying health states and health-related outcomes, and their determinants; and to establish a common language for describing health states that will permit comparison of data across countries, health care disciplines and services. Several efforts have been implemented to develop the use of ICF codes in geriatric care settings, improving appropriate qualifications for each code according to user interest (4). ICF has an important role in the clinical setting to identify the patient who requires multiple complex performance, and to evaluate the results of medical treatments, surgical, rehabilitative, palliative, undertaken in connection with such problem. At the same time, ICF takes an important role in the organizational-management, since based on the problems and strengths of the individual, being able to indicate the range of services appropriate to the care, treatment and rehabilitation (5).

In 2011 we processed and analysed, by the database of ANASTE (National Association of Nursing Home for Third Age) Calabria, data coming from 546 subjects resident in ANASTE's Nursing Homes. This study showed that $78.43 \%$ of the patients suffered from cognitive impairment whereas $52 \%$ had a severe degree of dementia. Was estimated, according to the ICD9 codes, that $65 \%$ of patients with dementia was suffering from Alzheimer's Disease (AD), whereas 23\% had vascular dementia (VD). The assessment of disability, by the ICF model for patients with dementia, allows us to formulate a dynamic functional-profile and identify the associations among health conditions, environmental and personal factors with disability levels.

\section{Methods}

A network of Long Term Care (LTC) Facilities, consisting of Nursing Homes and structures organization for Extensive Rehabilitation, is operating in the care of the frail elderly in Calabria. The access of these facilities is regulated according to the guidelines provided by the Calabria Region (DGR 685/2002, DGR 695/2003, LR 29/2008, DGR 3137/1999). This is an observational descriptive study carried out on a sample of residents, accross in two nursing homes associated to ANASTE
Calabria at June 2011. The present study was conducted by carrying out the customary practice of care, provided to all patients who belong to the LTC ANASTE Calabria, and nursing care didn't involve any different procedures. At the moment of admission in LTC the informed consent of the patients and/or their reference was acquired for daily care practice and use of their personal data. In brief, we used the National Institute of Neurological and Communicative Disorders and Stroke/Alzheimer's Disease and Related Disorders Association (NINCDS/ ADRA) (6) criteria for diagnosis of Alzheimer's disease (AD), and moreover the criteria of National Institute for Neurological Disorders and Stroke- Association Internationale puor la Recherche et Enseignement en Neusoscience (NINCDS-AIREN) (7) was used to diagnose vascular dementia. We enrolled 10 patients (7 F and 3M, mean age $69 \pm 17$ years) with probable AD (ADPr), according to the NINCDS-ADRDA criteria, and 10 patients ( $4 \mathrm{Fe}$ and $6 \mathrm{M}$, mean age $88 \pm 4.8$ years) affected by VD Probability (VDPr), according to NINDSAIREN criteria.

All patients underwent multidimensional geriatric assessment. The clinical diagnosis of dementia was firstly investigated by an interview through a detailed personal as well as family history and, subsequently, confirmed by the administration of psychometric tests. All patients fulfilled the criteria for dementia as described in the Diagnostic and Statistical Manual of Mental Disorders, Revised Fourth Edition (DSM IV) (8). The diagnosis of chronic pain was made according to ICD9-CM Official (9).The cognitive evaluation, was conducted by Folstein's Mini-Mental State Examination (MMSE) (10). According to MMSE, the patients were affected by severe, moderate or slight cognitive impairment, if MMSE score ranging 0-10, 11-20 or 21-23 respectively. The Clinical Dementia Rating (CDR) (11) was used for the staging of disease. The functional state was evaluated by the use of the Activity Daily Living scale (ADL) (12) and Barthel Index (BI) (13), in both scale a lower score indicate a worse of functional state. The burden of care was calculated in minutes assistance/day according to the Resource Utilization Group (RUG) III (14). The profiles of disability, by ICF, has been expressed in terms of Capacity and Performance. The Performance qualifier indicates the degree of Participation and/or Restriction in describing the current performance of the people in a task or action in their real environment. This context includes the environmental factors (physical, social and attitudinal) that can be coded using the Environmental Factors. Performance can be understood as «involvement in a life situation» or «lived experience» of people in the actual context in which they live. The Capacity qualifier indicates the degree of limitation by describing the person's ability to perform a task or an action. The Capacity qualifier focuses on limitations that are inherent or intrinsic features of the people themselves, due to the state of health of the person, without assistance of 
environmental factors (15). The components of the ICF classification are indicated with the letters b (body functions), s (body structures), d (dimension Activity/ Partecipation) and e (environmental factors) and are followed by a four-digit numeric code. The ICF also provides a scale of qualification for generic categories, where 0 stands for «no problem» (0-4\% limitation/ impairment), 1 for «sensitive issue» (5-24\% limitation/ impairment), 2 for «moderate problem» $(25-49 \%$ limitation/impairment), 3 for «serious problem» (50-95\% limitation / impairment), and 4 for «complete problem» (96-100\% limitation/impairment) (1). According to th ICF guidellnes, only the explicit and specific information were coded, such as those observed by the operator, through the patient's behavior, and codified in the categories closest to the operation observed, regardless of the patient's health. For the «Functions of the body» of the ICF components and «Activities and Participation», were calculated the prevalence of impairments and limitations in both the $A D$ group and VD group. The disability were coded according to the following scale: mild (5-24\%), medium (25-49\%), severe $(50-95 \%)$, complete (96 to $100 \%)$. The environmental factors (personal and instrumental) were listed as able in Barrier or No barrier, facilitator or No Facilitator (15).

\section{Results}

Patients with ADPr exhibited a severe impairment of functional status (ADL: $0.9 \pm 1.5$ and BI: $19 \pm 24.8 \mathrm{IB}$ ), and an advanced clinical stage of dementia (CDR: $3.8 \pm 0.6$ ). Instead of patients with VDPr showed less impairment of functional status (ADL: $1.1 \pm 0,87$ and BI; $31.6 \pm 24.9$ ), but an advanced clinical stage of dementia too (CDR of $3.4 \pm 0.69$ ). The care burden calculated according RUG III indicated an increased need of care in minutes of care for patients with $\mathrm{AD} \operatorname{Pr}(327,2 \mathrm{~min} /$ day/one $)$ compared with patients with VD Pr (213 $\mathrm{min} /$ day/one) (Tab1). In patients with dementia, relevant information, those related to Mental Functions (b1.) and those related to restrictions in performance associated with Mental Functions, were found; in particular, the items relating to Communication (d3), Interaction and Relationships (d7), mobility (d4) and self-care (d5), civil and social life of the community (d9), while learning and applying knowledge (d1), home life (d6) and Area of life Main (d8) were not applicable in this patient population. The global and specific Mental Functions, Communication and Interpersonal Relationships were more reduced in patients with ADPr compared with VDPr (Fig.1, 2 and 3), whereas in this group there were no significant differences about mobility and personal care. The assessment of the ICF AP, according to the qualifier "Capacity», showed that $60 \%$ of ADPr patients and $40 \%$ of VDPr patients had a complete disability, while $40 \%$ of ADPr patients and 60\% of VDPr patients showed a severe disability. Instead of observation according to the ICF qualifier "Performance", which takes into account environmental factors, showed a improvement of disability in both groups (Fig.4). The following environmental factors have been encoded Facilitator: as E355 (health professionals, nurses, rehabilitation therapists), E360 (educators, social workers.) E110 (drugs) and E115 (devices).

Table 1

Population and measurement characteristics

\begin{tabular}{lcc}
\hline Subjects & $\begin{array}{c}\text { ADPr Patients } \\
(\mathbf{N})\end{array}$ & $\begin{array}{c}\text { VDPr Patients } \\
(\mathbf{N})\end{array}$ \\
\hline Males & 3 & 6 \\
Females & 7 & 4 \\
Average age (years) & $69 \pm 17$ & $88 \pm 4.8$ \\
MMSE & \multicolumn{2}{c}{6} \\
Severe Cognitive Impairment & 8 & \\
Moderate Cognitive Impairment & 2 & of the mean \\
Measure & Mean + standard error \\
& ADPr Patients & VDPr Patients \\
CDR & $3.8 \pm 0.6$ & $3.4 \pm 0.69$ \\
ADL & $0.9 \pm 1.5$ & $1.1 \pm 0,87$ \\
BI & $19 \pm 24.8$ & $31.6 \pm 24.9$ \\
Care need (minutes / day) & 327,2 min/ day & 213 min / day
\end{tabular}

Notes: Alzheimer Disease Probable (ADPr); Vascular Dementia Probable (VDPr); Mini Mental State Examination (MMSE); Clinical Dementia Rating (CDR); Activities of Daily Living (ADL); Barthel Index (BI) Care Need by Resource Utilization Groups-III.

\section{Figure 1}

Impairments in ADPr and VDPr patients relating to the chapters: Mental Functions in the ICF component of Body Fuctions

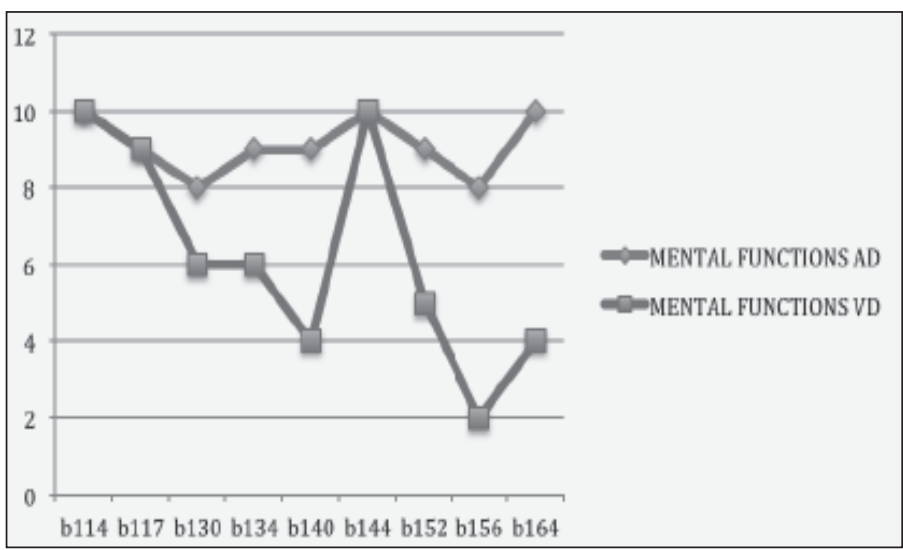

\section{Discussion}

The aim of this study is to describe the profiles of disability ICF across Activity and Partecipations domains in a cohort of residents suffering from ADPr and VDPr, 
to verify the effect of the environmental factors such as barrier or no barrier, facilitator or no facilitator and, finally, to sketch a checklist for the two forms of dementia. The ICF is based on a universal model that theoretically can be applied regardless of culture, age or care settings. The diagnosis alone does not explain what patients can do, what they need, and what can be the impairment of their functional status. The ICF allows us to obtain information on the operation of individuals and therefore plays an important role in the clinical setting to identify complicated patients, to evaluate the results of the medical treatment, rehabilitation and care. The ICF takes on an important role in the organizationalmanagement, indicating the range of services that are appropriate for each treatment (16). In Italian Nursing homes, all clinical activity and care given to patients with dementia, is planned by the multi-professional equipe, with a bio-psycho-social approach, through an operational tool called Personalized Care Plan (PCP). PCP is the main tool in the clinical-organizational pathways of care particularly for patients with dementia. The PCP developed through the use of ICF, can define goals, interventions, and related health professionals, for each detected need. The ICF, compared with other rating scales of disability and the burden of care, ADL, Barthel Index or RUGIII, has allowed to include the assessment of the environmental context of dementia patients: environmental factors such as nurses, rehabilitation therapists, educators, social workers, drugs, and devices in generally, that have proven to be important facilitators. Moreover, the use of the ICF has also permitted to plan the interventions of care for patients with dementia, and taking into account important aspects of daily life, usually less considered, such as communication, social relationships, recreation and free time. The ICF is a tool that allows to go beyond the negative focus of the impairment of functions and structures of the body, to assess the complexity of living with dementia. In the field of clinical management and about the quality of life for these patients, the latter task is of great importance.The present study, despite the small sample number, shows that the goals of care processed according to the indicators of Capacity and Performance, allow an improvement of disability in ADPr and VDPr patients. This study also allows us to identify one preliminary spectrum of problems, limitations and restrictions of the most common functioning and disability in these two patients groups. The identification of a specific checklist of disease, provides a more detailed description of the profiles of disability in the various forms of dementia and helps caregivers to deduce what interventions have to be made. There are different protocols for evaluation and classification systems of disability based on ICF for other chronic diseases, such as chronic widespread pain, osteoporosis, chronic ischemic heart disease, diabetes mellitus, obstructive pulmonary diseases, breast cancer, depression, and stroke (17). The
ICF core-sets contains the full spectrum of the problems of patients with a specific health condition and / or in a given clinical environment (18). It would be useful to also individuate a core-sets for the various forms of dementia, to improve the interventions and to have the possibility to verify the efficiency over a time period. The results of our study may be an initial contribution to this, but more researchs is needed on the development of the relevant ICF domains of this disease, especially taking into account other comorbidities such as metabolic and cardiovascular problems. The interventions, based on the ICF model, are able to orient the assistance and rehabilitation programs, improving the activities and participation in dementia patients, this may also induce a positive effect on their cognitive impairment and their quality of life.

\section{Figure 2}

Limitation or restriction in ADPr and VDPr patients, relating to the chapters: Comunications in the ICF component 'Activities and Participation'

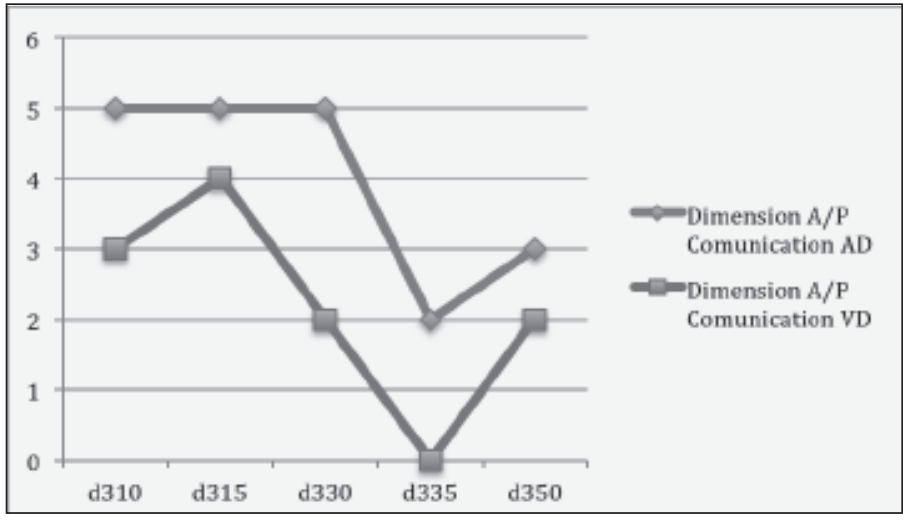

Figure 3

Limitation or restriction in ADPr and VDPr patients, relating to the chapters: Interpersonal Interactions and Relationship in the ICF component 'Activities and Participation'

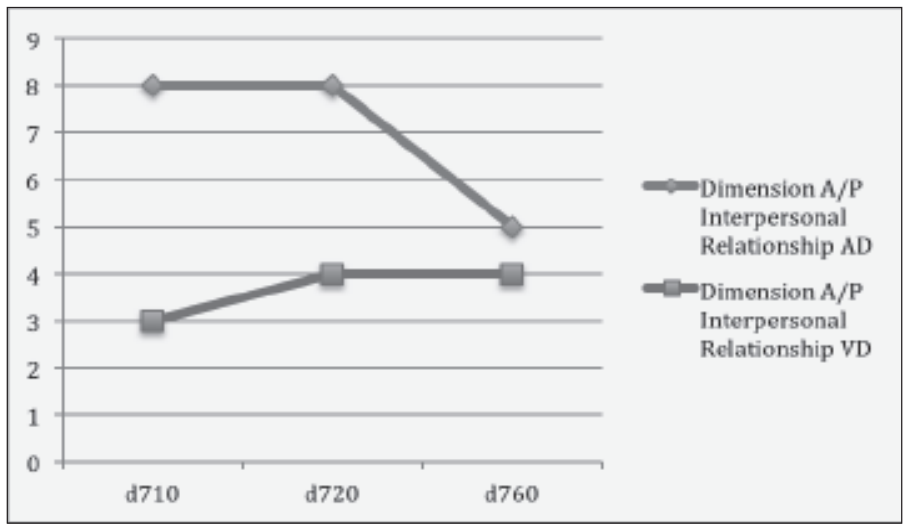


Figure 4

Prevalance of ICF disability in ADPr e VDPr patients according to the qualifier Capacity and Performance

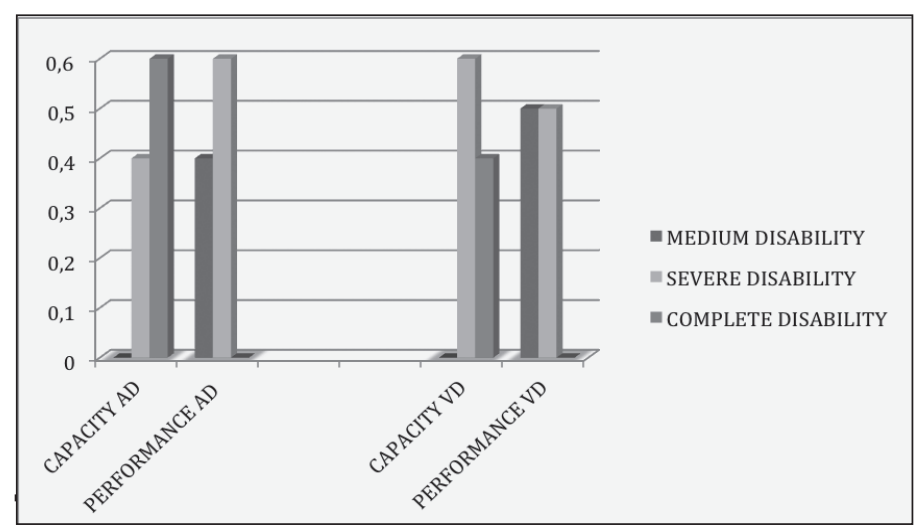

Acknowledgments: We thank the following Nursing Homes, for the recruitment of patients: RSA "San Domenico", Lamezia Terme (CZ) Italy, RSA "Villa Elisabetta" Cortale, (CZ) Italy, RSA “Ippolito Dodaro”, Falerna (CZ) Italy.

Funding: This study did not receive any funding for its implementation.

Disclosure: The authors report no conflicts of interest in this work.

Ethics Standards: The experiments described in this manuscript comply with the current Italian laws.

\section{References}

1. World Health Organization.: International Classification of Functioning, Disability and Health : ICF. Geneva, World Health Organization; 2001.

2. Ustun B: The international classification of functioning, disa- bility and health-a common framework for describing health states. 2002. http:/ whqlibdoc.who.int/publications / 2002/9241545518_Chap7.3.pdf.

3. Mueller M, Lohmann S, Strobl R, Boldt C, Grill E. Patients' functioning as predictor of nursing workload in acute hospital units providing rehabilitation care: a multi-centre cohort study. BMC Health Services Research 2010; 10:295307
4. Okochi J, Utsunomiya S, Takahashi T. Health measurement using the ICF: Test-retest reliability study of ICF codes and qualifiers in geriatric care. Health and Quality of Life Outcomes 2005; 3:46-59

5. Mueller M, Boldt C, Grill E, Strobl R, Stucki G: Identification of ICF categories relevant for nursing in the situation of acute and early post- acute rehabilitation. BMC Nurs 2008; 7:3.

6. McKhann G, Drachman D, Folstein M, Katzman R, Price D, Stadlan EM. Clinical diagnosis of Alzheimer's disease: report of the NINCDS-ARDRA Work Group under the auspices of Department of Health and Human Services Task Force on Alzheimer's Disease. Neurology. 1984;34:934-994.

7. Roman GC, Tatemichi TK, Erkinjuntti T, Cummings JL, Masdeu JC, Garcia $\mathrm{JH}$, et al. Vascular dementia: diagnostic criteria for research studies. Report of the NINDS-AIREN Inter- national Workshop. Neurology. 1993; 43(2):250-60.

8. American Psychiatric Association. Diagnostic and Statistical Manual of Mental Disorders, Fourth Edition, Text Revision (DSM-IV-TR). 2000. Washington, DC

9. ICD-9-CM Official Guidelines for Coding and Reporting Effective October 1, 2011

10. Folstein MF, Folstein SE, McHugh PR. "Mini-mental state." A practical method for grading the cognitive state of patients for the clinician. J Psychiatr Res. 1975;12:189-198.

11. Morris, J.C. The Clinical Dementia Rating (CDR): Current vision and scoring rules Neurology, 1993; 43:2412-2414

12. Katz S. Assessing self-maintenance: activities of daily living, mobility, and instrumental activities of daily living. J Am Geriatr Soc. 1983;31: 721-727.

13. Mahoney FI, Barthel DW. Functional evaluation: the Barthel Index. Md State Med J. 1965;14:61-65.

14. Carpenter I, Perry M, Challis D, Hope K. Identification of registered nursing care of residents in English nursing homes using the Minimum Data Set Resident Assessment Instrument (MDS/RAI) and Resource Utilisa- tion Groups version III (RUG-III). Age Ageing. 2002;32: 279-285.

15. World Health Organization. ICF Checklist Version 2.1a, Clinician Form for International Classification of Functioning, Disability and Health. September 2003. Available from: http://www.who.int/entity/classifications/icf/ training/icfchecklist.pdf.

16. Stier-Jarmer M, Grill E, Muller M, Strobl R, Quittan M, Stucki G. Validation of the comprehensive ICF Core Set for patients in geriatric post-acute rehabilitation facilities. Journal of rehabilitation medicine : official journal of the UEMS European Board of Physical and Rehabilitation Medicine. 2011;43(2):102-12.

17. Jelsma J. Use of the International Classification of Functioning, Disability and Health: a literature survey. Rehabil Med 2009; 41: 1-12

18. Cieza A, Ewert T, T. Berdirhan Ustun TB, Chatterji S, Kostanjsek N, Stucki G. Development of ICF core sets for patients with chronic condition. J Rehabil Med 2004; Suppl. 44: 9-11 